\title{
AUTHOR INDEX VOLUME 19
}

Abd-Rahman, MK, Selvakennedy, S \& Ahmad, H, Numerical Modeling of EDFL and Brillouin Erbium Fiber Laser

19 (2010) 281

Abdullah, KK, see Subha, PA

Acosta, G, see Lee, C-C

Agarwal, A, see Richardson, $K$

Ahmad, H, Zulkifli, MZ, Latif, AA \& Harun, SW, O-Band Multi-Wavelength Fiber Laser

19 (2010) 459

19 (2010) 767

19 (2010) 75

Ahmad, H, see Abd-Rahman, $M K$

Ahmad, H, see Harun, $S W$

Ahmad, H, see Shahi, $S$

Akazaki, T, see Ito, $H$

Alam, SU, see Harun, $S W$

Alkaisi, MM, see Chen, L

Amekura, H, see Plaksin, $O A$

Anderson, T, see Richardson, $K$

Asadpour, SH, see Sahrai, $M$

Asakawa, K, see Kumar, VD

Asakawa, K, see Watanabe, $Y$

Atiwongsangthong, N, Niemcharoen, S \& Titiroongruang, W,

Nanoporous Silicon Metal-Semiconductor-Metal Photodetector

Barati, M, see Rezaei, G

Beckers, T, Bittkau, K \& Carius, R, Investigation of Trapped Light in Thin-Film Silicon Solar Cells

Ben, PV, see Thi, TM

Bhardwaj, A, see Kumar, VD

Biswas, A, see Green, P

Bittkau, K, see Beckers, T

Blair, J, see Summers, CJ

Blinov, LM, Density of States and Lasing Threshold in Dye-Doped

Cholesteric Liquid Crystals

Botek, E, see de Meulenaere, E

Bratkovsky, A, see Holmström, P

Broer, DJ, see Urbach, HP

Bubeck, C, see Jahja, $M$

Bunch, JS, see Lee, $C$-C

Buy, C, see Lebrun, $S$

Cai, J, Lim, PH, Ishikawa, Y \& Wada, K, Silicon Waveguide Sidewall Smoothing by Resist Reflowing

19 (2010) 229

19 (2010) 281

19 (2010) 203

19 (2010) 123

19 (2010) 563

19 (2010) 203

19 (2010) 761

19 (2010) 737

19 (2010) 75

19 (2010) 503

19 (2010) 517

19 (2010) 543

19 (2010) 713

19 (2010) 131

19 (2010) 645

19 (2010) 237

19 (2010) 517

19 (2010) 339

19 (2010) 645

19 (2010) 489

19 (2010) 15

19 (2010) 1

19 (2010) 595

19 (2010) 189

19 (2010) 269

19 (2010) 767

19 (2010) 101

19 (2010) 801 
Cai, JH, Satoh, N, Yanagida, M \& Han, LY, Successive Large Perturbation Method for the Extraction of More Accurate Equivalent-Circuit-Parameters in Solar Cells

19 (2010) 637

Calderón, OG, see Ezquerro, JM

19 (2010) 153

Carius, R, see Beckers, $T$

Carlie, N, see Richardson, $K$

Chamorro-Posada, P, see Christian, JM

Champagne, B, see de Meulenaere, $E$

Chan, M-Y, see Lai, $S$ - $L$

Chen, L, Alkaisi, MM \& Liao, M-Y, Light Trapping Technique for Dye Sensitized Solar Cell

19 (2010) 645

19 (2010) 75

19 (2010) 389

19 (2010) 1

19 (2010) 603

19 (2010) 761

Chen, Y, Jaakola, J, Säynätjoki, A, Tervonen, A \& Honkanen, S, SERS-active Silver Nanoparticles in Ion-exchanged Glass

Chen, ZC, Hong, MH \& Chong, TC, Fabrication and Characterization of Broadband Terahertz Wire-Grid Polarizer

Choi, YG, Enhancing Emission Properties of Rare Earth Ions in Chalcogenide Glass Via Minute Compositional Adjustments

Chong, TC, see Chen, ZC

Christian, JM, McDonald, GS \& Chamorro-Posada, P, Helmholtz Solitons in Optical Materials with a Dual Power-Law Refractive Index

Clays, K, see de Meulenaere, E

Crne, M, see Summers, CJ

Cui, Y, see $L u, C$

Cui, Y, see $X u, S$

Das, M, Rana, S \& Sen, P, Second Harmonic Generation in ZnO Nanorods

Delaye, P, see Lebrun, $S$

Deparis, O, see Summers, CJ

Ding, YJ, Jiang, Y, Xu, G \& Zotova, IB, Recent Progress on Parametric Generation of Monochromatic THz Pulses

Echevarría, L, see Mendoza-Garía, A

Elborg, M, see Noda, T

Ezquerro, JM, Hernández, G, Nombela, M, Calderón, OG \& Melle, S, Fast Light Enhancement by Bidirectional Pumping in Erbium-Doped Fibers

Fan, S, Zhu, J, Shen, D, Gu, Y, Gu, X \& Luo, D, Tunable Transmission Using Azo-Dye-Doped Amorphous Polymer Films

Fang, Z, Hong, Q, Wang, C \& Zhu, X, Plasmonic Focusing Based on CdS Nanoribbon

Fiore, A, see Petti, L

Frey, R, see Lebrun, $S$

Fujii, A, see Fujiwara, $Y$

Fujii, M, see Yamaguchi, $K$

Fujimori, T, see Yamaki, $Y$

Fujiwara, Y, Murata, K, Ojima, M, Ogawa, Y, Kubo, H, Yoshida, H, Fujii, A \& Ozaki, M, Surface Plasmon Enhanced Photoluminescence on Biharmonic Grating Structure

19 (2010) 527

$19(2010) 827$

19 (2010) 663

19 (2010) 827

$19(2010) 389$

19 (2010) 1

19 (2010) 489

19 (2010) 327

19 (2010) 695

19 (2010) 445

19 (2010) 101

19 (2010) 489

19 (2010) 33

19 (2010) 427

19 (2010) 681

19 (2010) 153

19 (2010) 471

19 (2010) 729

19 (2010) 111

19 (2010) 101

19 (2010) 571

19 (2010) 811

19 (2010) 653

Fukuda, M, see Yamaguchi, K

Fukumi, K, see Kitamura, $N$ 
Furuya, K, see Ito, $H$

Gaillot, DP, see Summers, CJ

Green, L, see Patel, D

Green, P, Milovic, D, Sarma, AK, Lott, DA \& Biswas, A, Dynamics of Super-Sech Solitons in Optical Fibers

$\mathrm{Gu}, \mathrm{B}$, see $L u, C$

$\mathrm{Gu}, \mathrm{X}$, see Fan, $S$

Gu, Y, see Fan, $S$

Gumen, L, Nagaraj, Neogi, A \& Krokhin, A, Long-Range Surface Plasmons on Highly Anisotropic Dielectric Substrates

Han, L, see Peng, W

Han, LY, see Cai, JH

Han, W-T, see Ju, S

Han, W-T, see Kim, Y

Hao, F, Wang, R \& Wang, J, Focusing Control Based on SPPs-Scattering Modulation

Haraguchi, M, see Okamoto, $H$

Harun, SW, Ahmad, H, Lim, EL, Alam, SU \& Richardson, DJ, Single Mode Erbium Ytterbium-Doped Fiber Laser with Multimode Pumping

Harun, SW, see Ahmad, H

Harun, SW, see Shahi, $S$

Hashima, H, see Kitamura, $N$

Hernández, G, see Ezquerro, JM

Hidaka, T, see Kitamura, $N$

Hoan O, B, see Lee, H-S

Hoekstra, HJWM, see Uranus, HP

Holmström, P, Thylén, L \& Bratkovsky, A, Metal-Nanoshell/Quantum-Dot Array Waveguides with Compensated Loss

Hong, MH, see Chen, ZC

Hong, Q, see Fang, Z

Honkanen, S, see Chen, $Y$

$\mathrm{Hu}, \mathrm{J}$, see Richardson, $K$

Huang, W-P, see Wang, $L$

Iguchi, F, see Maegami, $Y$

Ikeda, $\mathrm{N}$, see Watanabe, $Y$

Ikeda, T, see Kitamura, $N$

Imai, T, see Nishide, J-I

Inami, W, see Matsumura, $Y$

Irokawa, Y, see Matsuki, $N$

Ishikawa, Y, see Cai, J

Ito, H, Furuya, K, Shibata, Y, Ootuka, Y, Nomura, S, Kashiwaya, S,

Yamaguchi, M, Tamura, H \& Akazaki, T, Mapping of Quantum-Hall Edge Channels by a Dilution-Refrigerator Based

Near-Field Scanning Optical Microscope

Jaakola, J, see Chen, $Y$

Jahja, M \& Bubeck, C, Nonlinear Optical Waveguide Spectroscopy of Poly(3-Butylthiophene)

Jeong, S, see Ju, S
19 (2010) 563

19 (2010) 489

19 (2010) 295

19 (2010) 339

19 (2010) 327

19 (2010) 471

19 (2010) 471

19 (2010) 613

19 (2010) 673

19 (2010) 637

19 (2010) 791

19 (2010) 781

19 (2010) 535

19 (2010) 583

19 (2010) 203

19 (2010) 229

19 (2010) 123

19 (2010) 753

19 (2010) 153

19 (2010) 753

19 (2010) 551

19 (2010) 255

19 (2010) 595

19 (2010) 827

19 (2010) 729

19 (2010) 527

19 (2010) 75

19 (2010) 319

19 (2010) 687

19 (2010) 543

19 (2010) 753

19 (2010) 629

19 (2010) 745

19 (2010) 703

19 (2010) 801

19 (2010) 563

19 (2010) 527

19 (2010) 269

19 (2010) 791 
Jeong, S, see Kim, Y

19 (2010) 781

Jessup, M, see Patel, D

Ji, X, Jiang, Q \& Liu, J, Incoherently Coupled Screening-Photovoltaic

19 (2010) 295

Spatial Soliton Pairs in Biased Two-Photon Photovoltaic

Photorefractive Crystals

Jiang, G-C, see Wang, $Y-L$

Jiang, Q, see Ji, $X$

Jiang, Y, see Ding, YJ

Jo, M, see Kawazu, T

Joseph, A \& Porsezian, K, Periodic Wave Solutions to Modified Nonlinear Schrödinger Equation Pertaining to Negative Index Materials

Ju, S, Watekar, PR, Jeong, S, Kim, Y \& Han, W-T, Nonlinear Optical Properties of Zinc Doped Germano-Silicate Glass Optical Fiber

$\mathrm{Ju}, \mathrm{S}$, see Kim, $Y$

Kaivola, M, see Priimagi, A

Kajihara, Y, Kosaka, K \& Komiyama, S, Passively Detecting Thermal Evanescent Waves from Room Temperature Objects

Kandiah, K, see Menon, PS

Karasawa, N, see Tada, K

Kashiwaya, S, see Ito, $H$

Kauranen, M, see Priimagi, A

Kawabe, Y, see Nishide, J-I

Kawata, Y, see Matsumura, $Y$

Kawazu, T, Noda, T, Mano, T, Jo, M \& Sakaki, H, Effects of Antimony Flux on Morphology and Photoluminescence Spectra of GaSb Quantum Dots Formed on GaAs by Droplet Epitaxy

19 (2010) 167

19 (2010) 407

19 (2010) 167

19 (2010) 33

19 (2010) 819

$19(2010) 177$

19 (2010) 791

19 (2010) 781

19 (2010) 57

19 (2010) 589

19 (2010) 209

19 (2010) 723

19 (2010) 563

19 (2010) 57

19 (2010) 629

19 (2010) 745

Kim, Y, Ju, S, Jeong, S \& Han, W-T, Photonic Properties of Ti Doped Optical Fiber

Kim, Y, see Ju, $S$

Kimerling, L, see Richardson, $K$

Kimura-Suda, $\mathrm{H}$, see Nishide, $J-I$

King, N, see Patel, D

Kishimoto, N, see Plaksin, $O A$

Kitagawa, Y, see Watanabe, $Y$

Kitamura, N, Fukumi, K, Nakamura, J, Hidaka, T, Ikeda, T,

Hashima, H \& Nishii, J, Low-Tg Bismuth Phosphate Glasses for Glass-Imprinting and Fabrication of $2 D$ Sub-Wavelength Structure

Komiyama, S, see Kajihara, $Y$

Kono, K, see Plaksin, OA

Kosaka, K, see Kajihara, $Y$

Krokhin, A, see Gumen, L

Kubo, H, see Fujiwara, $Y$

Kumar, VD, Bhardwaj, A, Mishra, D \& Asakawa, K, Directional and Polarization Properties of a Plasmonic Cross Nanoantenna

Kuriakose, VC, see Subha, PA

Kuzyk, MG, see Szafruga, UB

19 (2010) 819

19 (2010) 781

19 (2010) 791

$19(2010) 75$

19 (2010) 629

19 (2010) 295

19 (2010) 737

19 (2010) 543

19 (2010) 753

19 (2010) 589

19 (2010) 737

19 (2010) 589

19 (2010) 613

19 (2010) 571

19 (2010) 517

19 (2010) 459

19 (2010) 379

Lai, S-L, Chan, M-Y, Tong, Q-X, Lo, M-F, Ng, T-W, Lee, C-S \& Lee, $\mathrm{S}-\mathrm{T}$, Color Tunable Electroluminescence from Organic 
Light-Emitting Devices by Manipulating Exciton and Exciplex Emissions

Latif, AA, see Ahmad, $H$

Lebrun, S, Buy, C, Delaye, P, Frey, R, Pauliat, G \& Roosen, G, Optical Characterizations of a Raman Generator Based on a Hollow Core Photonic Crystal Fiber Filled with a Liquid

Lee, C-C, Schibli, TR, Acosta, G \& Bunch, JS, Ultra-Short Optical Pulse Generation with Single-Layer Graphene

Lee, C-S, see Lai, $S$ - $L$

Lee, E-H, see Lee, $H-S$

Lee, H-S, Song, J-H, Hoan O, B, Lee, SG \& Lee, E-H, Modal Characteristics of Plasmonic Nanostrip Waveguides and Their Use for Functional Devices

19 (2010) 603

19 (2010) 229

Lee, SG, see Lee, $H-S$

Lee, S-T, see Lai, $S$ - $L$

Li, C, see Zhang, $B$

Li, M, see Wang, $Y$-L

Liao, M-Y, see Chen, $L$

Lim, EL, see Harun, $S W$

Lim, PH, see Cai, $J$

Liu, J, see Ji, $X$

Lo, M-F, see Lai, $S-L$

Lott, DA, see Green, $P$

Lu, C, Cui, Y, Gu, B \& Wang, F, Study on the Refractive Non-Linearity of Three-Photon Absorbing Media with the Z-Scan Technique

Luo, D, see Fan, $S$

Luzinov, I, see Richardson, $K$

Maegami, Y, Iguchi, F \& Yugami, H, Surface Structural Design of Selective Emitter for Methane Steam Reforming

Manirahulan, K, see Vinitha, G

Manna, L, see Petti, L

Mano, T, see Kawazu, T

Mano, T, see Noda, T

Marumoto, K, see Yamaki, $Y$

Matsuki, N, Nakano, Y, Irokawa, Y \& Sumiya, M, Heterointerface Properties of Novel Hybrid Solar Cells Consisting of Transparent Conductive Polymers and III-Nitride Semiconductor

19 (2010) 101

19 (2010) 767

19 (2010) 603

19 (2010) 551

19 (2010) 551

19 (2010) 551

19 (2010) 603

19 (2010) 415

19 (2010) 407

19 (2010) 761

19 (2010) 203

19 (2010) 801

19 (2010) 167

19 (2010) 603

19 (2010) 339

19 (2010) 327

19 (2010) 471

19 (2010) 75

19 (2010) 687

19 (2010) 621

19 (2010) 111

19 (2010) 819

19 (2010) 681

19 (2010) 653

19 (2010) 703

Matsumura, Y, Inami, W \& Kawata, Y, Laser Light Control of Self-Organization Process

McDonald, GS, see Christian, JM

Melle, S, see Ezquerro, JM

Mendoza-Garía, A, Romero-Depablos, A, Ortega, MA, Paz, JL \& Echevarría, L, Theoretical Model for the Calculation of Optical Properties of Gold Nanoparticles

19 (2010) 745

19 (2010) 389

19 (2010) 153

19 (2010) 427

Menon, PS, Kandiah, K \& Shaari, S, Variation of $M Q W$ Design Parameters in a GaAs/InP-Based LW-VCSEL and Its Effects on the Spectral Linewidth

de Meulenaere, E, de Wergifosse, M, Botek, E, Spaepen, S, Champagne, B, Vanderleyden, J \& Clays, K, Nonlinear Optical 
Properties of mStrawberry and mCherry for Second Harmonic Imaging

19 (2010) 1

Milovic, D, see Green, P

Minh, NV \& Thang, DV, Multiferroic Materials $\mathrm{Bi}_{1-x} \mathrm{Sm}_{x} \mathrm{FeO}_{3}: A$

19 (2010) 339

Study of Raman and Absorption Spectroscopies

Minh, NV, see Phuong, DTT

Mishra, D, see Kumar, VD

Mitatha, S, see Udomariyasap, $P$

Mitatha, S, see Youplao, $P$

Mitsuishi, K, see Noda, T

Moghaddam, MRA, see Shahi, $S$

Mori, T, see Yamaki, Y

Mormile, P, see Petti, L

Murata, K, see Fujiwara, $Y$

Nagaraj, see Gumen, L

Nakamura, J, see Kitamura, $N$

Nakano, Y, see Matsuki, $N$

Nakao, M, Yamaguchi, M \& Yabu, S, Imprint-Mold-Cleaning by Vacuum Ultraviolet Light

Neogi, A, see Gumen, $L$

$\mathrm{Ng}, \mathrm{T}-\mathrm{W}$, see Lai, $S-L$

Niemcharoen, S, see Atiwongsangthong, $N$

Nishide, J-I, Kimura-Suda, H, Imai, T, Sasabe, H \& Kawabe, Y, Photorefractive Polymer with High Optical Gain Under Non-Electric Field

19 (2010) 247

19 (2010) 219

19 (2010) 517

19 (2010) 303

19 (2010) 371

19 (2010) 681

19 (2010) 123

19 (2010) 653

19 (2010) 111

19 (2010) 571

19 (2010) 613

19 (2010) 753

19 (2010) 703

19 (2010) 773

19 (2010) 613

19 (2010) 603

19 (2010) 713

Nishii, J, see Kitamura, $N$

Noda, T, Mano, T, Elborg, M, Mitsuishi, K \& Sakoda, K, Fabrication of a GaAs/AlGaAs Lattice-Matched Quantum Dot Solar Cell

Noda, T, see Kawazu, T

Nombela, M, see Ezquerro, JM

Nomura, S, see Ito, $H$

Noppanakeepong, S, see Udomariyasap, $P$

Norizan, SF, see Shahi, $S$

Ogawa, Y, see Fujiwara, $Y$

Ojima, M, see Fujiwara, $Y$

Okamoto, H, Yamaguchi, K, Haraguchi, M \& Okamoto, T, Plasmonic Racetrack Resonator for Application to Photonic Integrated Circuits at Sub-Wavelength

Okamoto, T, see Okamoto, $H$

Ootuka, Y, see Ito, $H$

Ortega, MA, see Mendoza-Garía, A

Ozaki, M, see Fujiwara, $Y$

Ozaki, N, see Watanabe, $Y$

Park, JO, see Summers, CJ

Patel, D, Vance, C, King, N, Jessup, M, Green, L \& Sarkisov, S, Strong Visible Upconversion in Rare Earth Ion-Doped $\mathrm{NaYF}_{4}$ Crystals

$19(2010) 295$

Pauliat, G, see Lebrun, $S$

19 (2010) 101

Paz, JL, see Mendoza-Garía, A

19 (2010) 629

19 (2010) 753

19 (2010) 681

19 (2010) 819

19 (2010) 153

19 (2010) 563

19 (2010) 303

19 (2010) 123

19 (2010) 571

19 (2010) 571

19 (2010) 583

19 (2010) 583

19 (2010) 563

19 (2010) 427

19 (2010) 571

19 (2010) 543

19 (2010) 489

19 (2010) 427 
Peng, W, Yanagida, M \& Han, L, Rutile-Anatase $\mathrm{TiO}_{2}$ Photoanodes for Dye-Sensitized Solar Cells

Pereira, SF, see Urbach, HP

Petit, L, see Richardson, $K$

Petti, L, Rippa, M, Fiore, A, Manna, L \& Mormile, P, Dynamic Orientational Photo-Refractive Gratings Observed in CdSe/CdS Nanorods Doped Nematic Liquid Crystal Cells

Phattaraworamet, T, see Youplao, $P$

Phuong, DTT \& Minh, NV, Dopant Effects on the Structural, Optical and Electrical Transport Properties in $\mathrm{SrTi}_{1-x} \mathrm{Co}_{x} \mathrm{O}_{3}$ Nanoparticles Synthesized by Sol-Gel Method

19 (2010) 673

19 (2010) 189

19 (2010) 75

19 (2010) 111

19 (2010) 371

19 (2010) 219

19 (2010) 737

19 (2010) 177

Radiation Photonics: A Case of Metal-Nanoparticle Composites

Porsezian, K, see Joseph, A

Priimagi, A, Kaivola, M, Virkki, M, Rodríguez, FJ \& Kauranen, M, Suppression of Chromophore Aggregation in Amorphous Polymeric Materials: Towards More Efficient Photoresponsive Behavior

Ramalingam, A, see Vinitha, $G$

Rana, S, see Das, $M$

Rezaei, G, Vahdani, MRK \& Barati, M, Intersubband Optical Absorption Coefficients and Refractive Index Changes of an Ellipsoidal Finite Potential Quantum Dot

Richardson, DJ, see Harun, $S W$

Richardson, K, Petit, L, Carlie, N, Zdyrko, B, Luzinov, I, Hu, J, Agarwal, A, Kimerling, L, Anderson, T \& Richardson, M, Progress on the Fabrication of On-Chip, Integrated Chalcogenide Glass (CHG)-Based Sensors

Richardson, M, see Richardson, $K$

Rippa, M, see Petti, L

Rodríguez, FJ, see Priimagi, A

Romero-Depablos, A, see Mendoza-Garía, A

Roosen, G, see Lebrun, $S$

Sadighi, R, see Sahrai, $M$

Sahrai, M, Asadpour, SH \& Sadighi, R, Enhanced Kerr Nonlinearity in a Four-Level EIT Medium

Sakaki, H, see Kawazu, T

Sakoda, K, see Noda, T

Sarkisov, S, see Patel, D

Sarma, AK, see Green, $P$

Sasabe, H, see Nishide, J-I

Satoh, N, see Cai, JH

Säynätjoki, A, see Chen, $Y$

Schibli, TR, see Lee, $C$-C

Selvakennedy, S, see Abd-Rahman, $M K$

Sen, $\mathrm{P}$, see Das, $M$

Shaari, S, see Menon, PS

Shahi, S, Harun, SW, Norizan, SF, Moghaddam, MRA \& Ahmad, H, Brillouin-Raman Multi-Wavelength Laser Comb Generation Based on Bi-EDF by Using Dual-Wavelength in Dispersion Compensating Fiber
19 (2010) 57

19 (2010) 621

19 (2010) 445

19 (2010) 131

19 (2010) 203

19 (2010) 75

19 (2010) 75

19 (2010) 111

19 (2010) 57

19 (2010) 427

19 (2010) 101

19 (2010) 503

19 (2010) 503

19 (2010) 819

19 (2010) 681

19 (2010) 295

19 (2010) 339

19 (2010) 629

19 (2010) 637

19 (2010) 527

19 (2010) 767

19 (2010) 281

19 (2010) 445

19 (2010) 209

19 (2010) 123 
Shen, D, see Fan, $S$

Shibata, Y, see Ito, $H$

Song, J-H, see Lee, H-S

Spaepen, S, see de Meulenaere, E

Srinivasarao, M, see Summers, CJ

Stoffer, R, see Uranus, HP

Subha, PA, Abdullah, KK \& Kuriakose, VC, Three-Dimensional

Light Bullets in Cubic-Quintic Media Stabilized by Periodic

Variation of Diffraction and Dispersion

Sugimoto, Y, see Watanabe, $Y$

Sumiya, M, see Matsuki, $N$

Summers, CJ, Gaillot, DP, Crne, M, Blair, J, Park, JO, Srinivasarao,

M, Deparis, O, Welch, V \& Vigneron, J-P, Investigations and

Mimicry of the Optical Properties of Butterfly Wings

Szafruga, UB, Kuzyk, MG \& Watkins, DS, Maximizing the

Hyperpolarizability of One-Dimensional Systems

Tada, K, Yamanaka, A \& Karasawa, N, Broadband Coherent Anti-Stokes Raman Scattering Microspectroscopy Using the Soliton Pulses from a Photonic Crystal Fiber - Observation of Raman

Line in Diamond Powders

Takata, Y, see Watanabe, $Y$

Takeda, Y, see Plaksin, $O A$

Tamura, H, see Ito, $H$

Teeka, C, see Youplao, $P$

Tervonen, A, see Chen, $Y$

Thang, DV, see Minh, NV

Thi, TM, Van, BH \& Ben, PV, Study of Microstructure and Optical Properties of PVA-Capped ZnS : Cu Nanocrystalline Thin Films

Thylén, L, see Holmström, $P$

Titiroongruang, W, see Atiwongsangthong, $N$

Tong, Q-X, see Lai, S-L

Udomariyasap, P, Noppanakeepong, S, Mitatha, S \& Yupapin, PP, THz Light Pulse Generation and Storage Within an Embedded Optical Waveguide System

Uranus, HP, Hoekstra, HJWM \& Stoffer, R, Modeling and Design of Realistic $\mathrm{Si}_{3} \mathrm{~N}_{4}$-Based Integrated Optical Programmable Power Splitter

Urbach, HP, Pereira, SF \& Broer, DJ, On Optimum Pupil Fields with Maximum Electric Field Component in Focus

Vahdani, MRK, see Rezaei, $G$

Van, BH, see Thi, TM

Vance, C, see Patel, D

Vanderleyden, J, see de Meulenaere, E

Vigneron, J-P, see Summers, CJ

Vinitha, G, Manirahulan, K \& Ramalingam, A, Optical Limiting Characteristics of Core-Shell Nanoparticles

Virkki, M, see Priimagi, A

Wada, K, see Cai, J

Wakaki, M \& Yokoyama, E, Dielectric Analysis on Optical Properties of $\mathrm{ZrO}_{2}$ Thin Films Dispersed with Silver Nanoparticles

19 (2010) 471

19 (2010) 563

19 (2010) 551

$19(2010) 1$

19 (2010) 489

19 (2010) 255

19 (2010) 459

19 (2010) 543

19 (2010) 703

19 (2010) 489

19 (2010) 379

19 (2010) 723

19 (2010) 543

19 (2010) 737

19 (2010) 563

19 (2010) 371

19 (2010) 527

19 (2010) 247

19 (2010) 237

19 (2010) 595

19 (2010) 713

19 (2010) 603

19 (2010) 303

19 (2010) 255

19 (2010) 189

19 (2010) 131

19 (2010) 237

19 (2010) 295

19 (2010) 1

19 (2010) 489

19 (2010) 621

19 (2010) 57

19 (2010) 801

19 (2010) 835 
Wang, C, see Fang, Z

Wang, C, see $X u, S$

Wang, C, see Zhang, $X$

Wang, F, see $L u, C$

Wang, J, see Hao, F

Wang, L, Wang, Z \& Huang, W-P, Comparison between Ultrafast Gain and Phase Dynamics in Semiconductor Optical Amplifiers

Wang, R, see Hao, F

Wang, Y-F, see Wang, $Y$ - $L$

Wang, Y-L, Jiang, G-C, Wang, Y-F, Zheng, J-J \& Li, M, Third-Order Nonlinear Optical Investigation of a Novel Material in the Near-IR Regions

Wang, Z, see Wang, L

Watanabe, Y, Ikeda, N, Takata, Y, Kitagawa, Y, Ozaki, N, Sugimoto, Y \& Asakawa, K, Wideband Operation of 2D Photonic Crystal

Directional Coupler with Topology Optimized Waveguide Bends

Watekar, PR, see Ju, S

Watkins, DS, see Szafruga, UB

Wazwaz, A-M, A Completely Integrable System of Coupled Modified KdV Equations

Welch, V, see Summers, CJ

de Wergifosse, M, see de Meulenaere, E

$\mathrm{Xu}, \mathrm{G}$, see Ding, YJ

$\mathrm{Xu}, \mathrm{S}$, Wang, C \& Cui, Y, Theoretical Characters of $M g X(\mathrm{X}=T e$, Se, $S$ and $O)$ Clusters

$\mathrm{Xu}, \mathrm{W}$, see Zhang, $B$

Yabu, S, see Nakao, $M$

Yamaguchi, K, Fujii, M \& Fukuda, M, A Structure for Coupling Whispering Gallery Mode with Surface Plasmons

Yamaguchi, K, see Okamoto, $H$

Yamaguchi, M, see Ito, $H$

Yamaguchi, M, see Nakao, $M$

Yamaki, Y, Marumoto, K, Fujimori, T \& Mori, T, Dependence of Device Characteristics of Bulk-Heterojunction Organic Thin-Film Solar Cells on Concentration of Glycerol and Sorbitol Addition in Pedot:PSS Solutions for Fabricating Buffer Layers

Yamanaka, A, see Tada, $K$

Yanagida, M, see Cai, JH

Yanagida, M, see Peng, $W$

Yang, Z-P \& Zhong, W-P, Novel Laguerre-Gaussian Beam in Strongly Nonlocal Nonlinear Media

Yang, Z-P, see Zhong, W-P

Yokoyama, E, see Wakaki, $M$

Yoshida, H, see Fujiwara, $Y$

Youplao, P, Phattaraworamet, T, Mitatha, S, Teeka, C \& Yupapin, PP, Novel Optical Trapping Tool Generation and Storage Controlled by Light

Yugami, H, see Maegami, Y

Yupapin, PP, see Udomariyasap, $P$

Yupapin, PP, see Youplao, P
19 (2010) 729

19 (2010) 695

19 (2010) 437

19 (2010) 327

19 (2010) 535

19 (2010) 319

19 (2010) 535

19 (2010) 407

19 (2010) 407

19 (2010) 319

19 (2010) 543

19 (2010) 791

19 (2010) 379

19 (2010) 145

19 (2010) 489

19 (2010) 1

19 (2010) 33

19 (2010) 695

19 (2010) 415

19 (2010) 773

19 (2010) 811

19 (2010) 583

19 (2010) 563

19 (2010) 773

19 (2010) 653

19 (2010) 723

19 (2010) 637

19 (2010) 673

19 (2010) 479

19 (2010) 311

19 (2010) 835

19 (2010) 571

19 (2010) 371

19 (2010) 687

19 (2010) 303

19 (2010) 371 
Zdyrko, B, see Richardson, $K$

$19(2010) 75$

Zeng, Y, see Zhang, $X$

19 (2010) 437

Zhang, B, Xu, W \& Li, C, Low-Power All-Optical Switching in a Nonlinear $M-Z$ Interferometer Made from a

Single-Nonlinear-Fiber-Connected LPFG Pair

19 (2010) 415

Zhang, J, see Zhang, $X$

Zhang, X, Wang, C, Zeng, Y \& Zhang, J, Nonlinear Refraction and

19 (2010) 437

Photoinduced Birefringence in the Methyl-Red-Doped Polymer Thin

Film

Zheng, J-J, see Wang, $Y$-L

Zhong, W-P \& Yang, Z-P, Spatial Solitary Waves in Generalized Non-Local Nonlinear Media

Zhong, W-P, see Yang, Z-P

Zhu, J, see Fan, $S$

Zhu, X, see Fang, $Z$

19 (2010) 437

19 (2010) 407

Zotova, IB, see Ding, $Y J$

Zulkifli, MZ, see Ahmad, H

19 (2010) 311

19 (2010) 479

19 (2010) 471

19 (2010) 729

19 (2010) 33

19 (2010) 229 